\title{
On how thick diffusers can contribute to the design of optical security systems
}

Kavan Ahmadi, Ignasi Juvells, Artur Carnicer

Kavan Ahmadi, Ignasi Juvells, Artur Carnicer, "On how thick diffusers can contribute to the design of optical security systems," Proc. SPIE 11207, Fourth International Conference on Applications of Optics and Photonics, 112071H (3 October 2019); doi: 10.1117/12.2527393

SDIE Event: IV International Conference on Applications of Optics and Photonics (AOP 2019), 2019, Lisbon, Portugal 


\title{
On how thick diffusers can contribute to the design of optical security systems
}

\author{
Kavan Ahmadi, Ignasi Juvells, Artur Carnicer \\ Universitat de Barcelona (UB), Facultat de Física, Departament de Física Aplicada, \\ Martí i Franquès 1, 08028 Barcelona (Catalunya, Spain)
}

\begin{abstract}
We recently reported that phase encoded samples produced with metallic components (e.g. gold nano-particles) can be distinguished by means of polarized light. Classification was carried out using data obtained from speckle distributions. Despite this approach is very successful, it cannot be used with codes made of materials that do not change the state of polarization of the illumination source. In the present communication we analyze the feasibility of using optical diffusers as polarizing phase encoders for optical security systems. Preliminary optical results seem to support our thesis.
\end{abstract}

Keywords: Optical security, polarization, optical diffusers

\section{INTRODUCTION}

Optical diffusers have been widely investigated from both theoretical and practical points of view. ${ }^{1}$ In particular, a large number of papers focus on numerical models related to the behavior of light interacting with such devices (see, for instance, ${ }^{2,3}$ ). Despite diffusers have been investigated from multiples points of view, polarization is not a particularly interesting property in the present analysis. ${ }^{4}$ The objective of this communication is to evaluate to what extent a thick diffuser modifies and reinforces the uniqueness of the optical signature of the sample. In order to achieve this objective, we develop a ray-tracing calculation in order to determine polarization changes; data from a real diffuser surface is used. Then, experimental results validate the proposed model.

Recent developments in optical authentication and validation demonstrate the ability of the properties of light to distinguish among counterfeit and true samples. ${ }^{5}$ Sometimes, metallic nanoparticles or thin films technology is used during the fabrication process in order to provide a strong polarimetric signature. In particular, the combined examination of the state of polarization of light after interacting with the sample and the statistical analysis of the speckle patterns provide enough information to train machine learning methods. In this way, these techniques would be able to predict whether the sample is true or fake. ${ }^{6-8}$ On the other hand, phase-encoding masks using cello-type diffusers provide an extra security layer. After propagation, phase encoded information becomes a Poisson-like noise distribution and thus, any attempt to access to the original signal is very difficult. In a recent paper we studied the capacity of three-dimensional phase coders using thick diffusers to enrich the amount of information for training machine learning algorithms. ${ }^{9}$

The paper is organized as follows. In section 2, we describe the numerical approach used and present several experimental results that validate the model. Finally, our conclusions are presented in section 3.

\section{NUMERICAL MODEL AND EXPERIMENTAL RESULTS}

In order to perform a ray-tracing calculation using real data, we measured the two-dimensional profile of one of the surfaces of a Polyvinyl Chloride (PVC) sample using a profilometer. The area considered is $0.637 \mathrm{~mm} \times 0.478$ $\mathrm{mm}$ and the peak to valley depth is $0.0247 \mu \mathrm{m}$. This surface is depicted in Fig. 1. We assume the refractive index is $n_{d}=1.539$.

We numerically estimated the Stokes parameters $S_{0}, S_{1}$ and $S_{2}$, when the diffuser is illuminated by a linearly polarized source at $45^{\circ}$ with respect to the $x$-axis. In the first case, the diffuser is placed normal to the propagation direction. Then, in order to emphasize the polarization effects, the angle of incidence is set to $45^{\circ}$. The results are presented in Fig. 2. As expected, $S_{2}$ is almost 1 . Nevertheless, $S_{1}$ displays small but non-negligible values in most of the pixels of the image

Fourth International Conference on Applications of Optics and Photonics, edited by

Manuel F. M. Costa, Proc. of SPIE Vol. 11207, 112071H · C) 2019 SPIE

CCC code: $0277-786 X / 19 / \$ 21 \cdot$ doi: $10.1117 / 12.2527393$

Proc. of SPIE Vol. $11207112071 \mathrm{H}-1$

Downloaded From: https://www.spiedigitallibrary.org/conference-proceedings-of-spie on 18 Oct 2019 Terms of Use: https://www.spiedigitallibrary.org/terms-of-use 


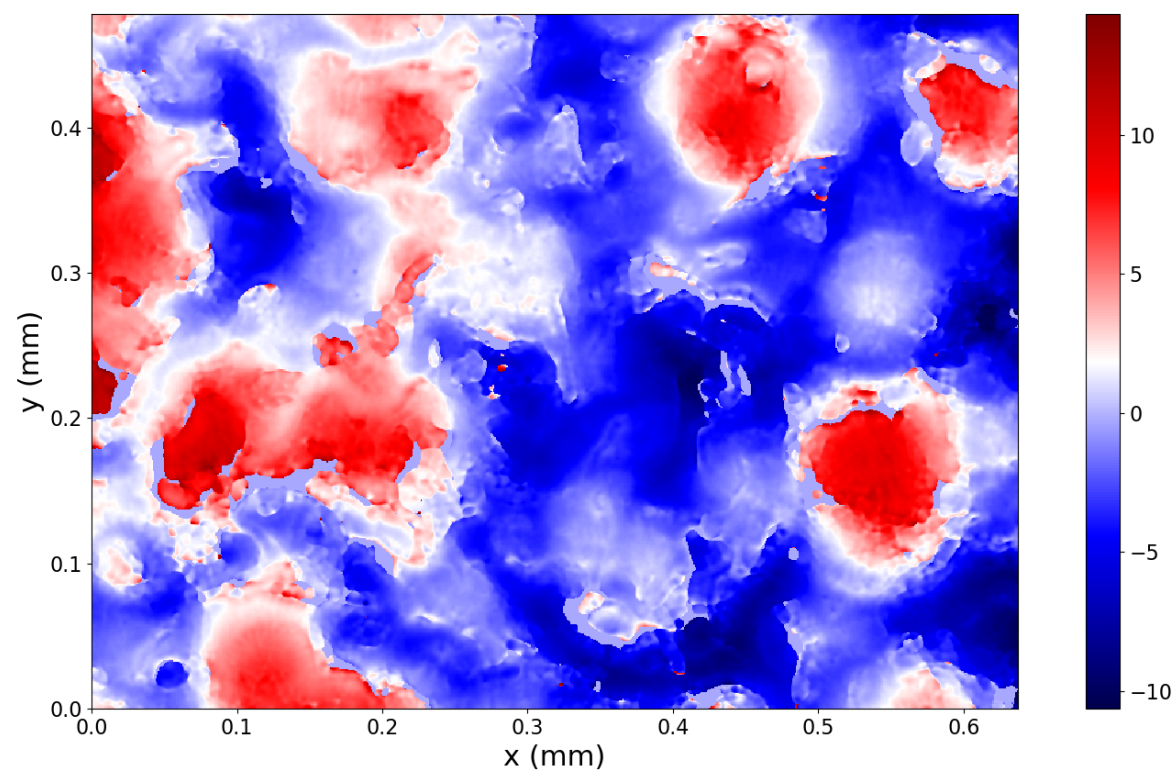

Figure 1. False color representation of the measured surface. The scale of the colorbar is in microns.

so

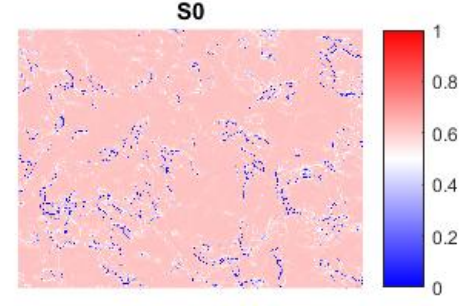

so

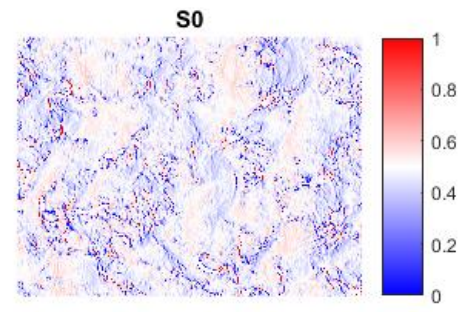

S1

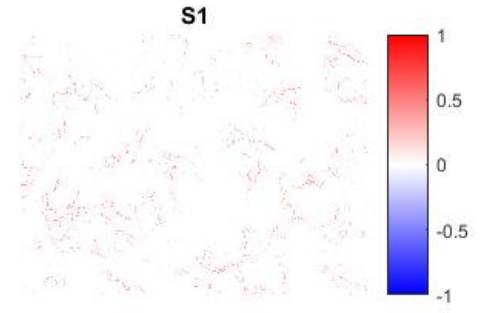

S1

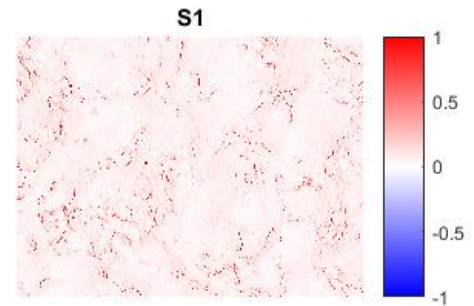

S2

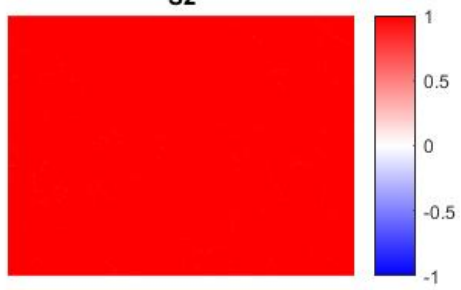

S2

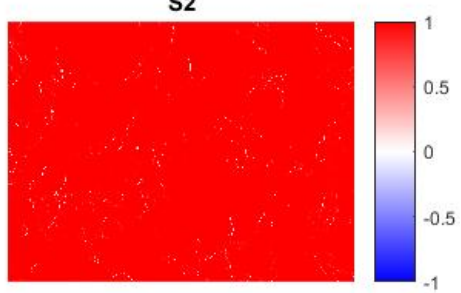

Figure 2. Numerical estimation of the Stokes parameters after the surface. First row: angle of incidence $0^{\circ}$. Second row: angle of incidence $45^{\circ}$.

We performed a simple experiment in order to measure the Stokes parameters of the diffuser. Figure 3 shows a picture of the optical setup. A linearly polarized beam $\left(45^{\circ}\right.$ with respect to the $x$-axis) illuminates the sample. Then, the Speckle distributions are recorded by a CCD camera. The diffuser used for the tests is a Thorlabs N-BK7 Ground Glass Difusser, 120 Grit.

Figure 4 displays the Stokes parameters $S_{0}, S_{1}$ and $S_{2}$ when the diffuser is placed at $0^{\circ}$ and $45^{\circ}$ with respect to the propagation beam. As expected $S_{2}$ is close to one but $S_{1}$ is not zero in several points of the image. Note that these results are compatible with the simulations presented in Fig. 2. 


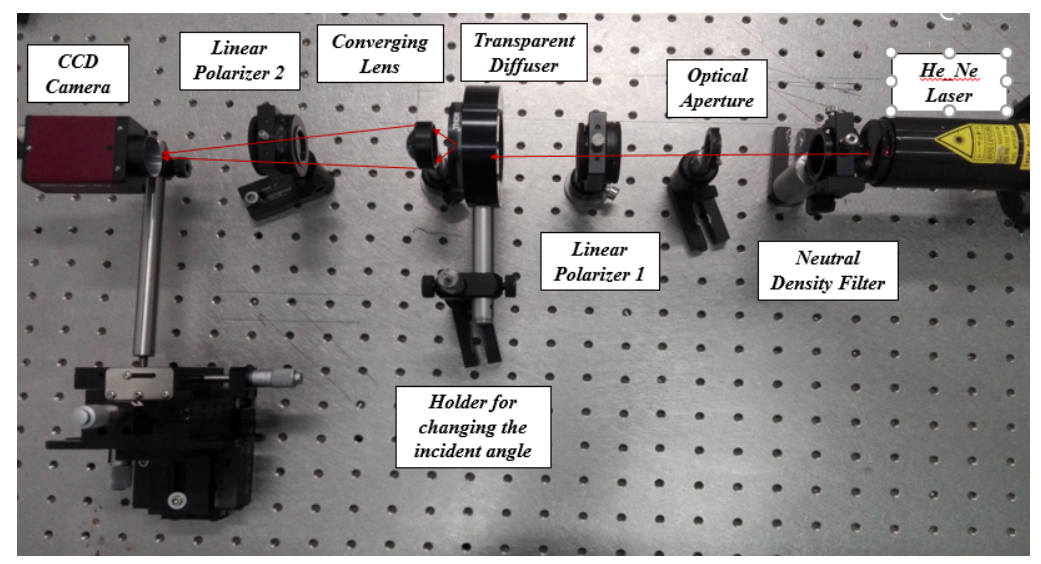

Figure 3. Optical setup.

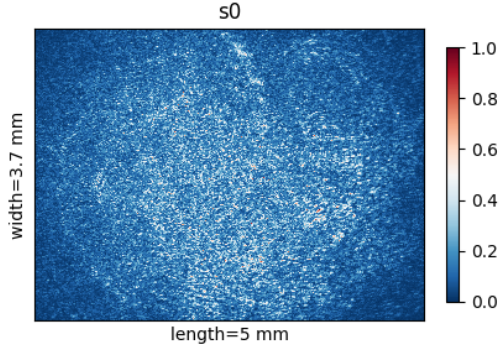

so

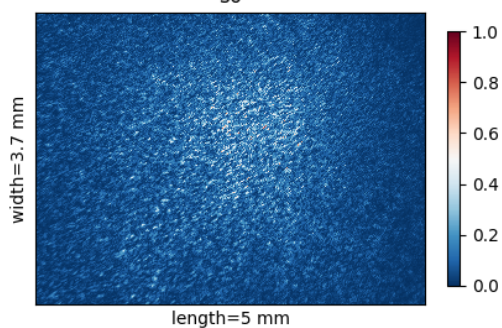

s1

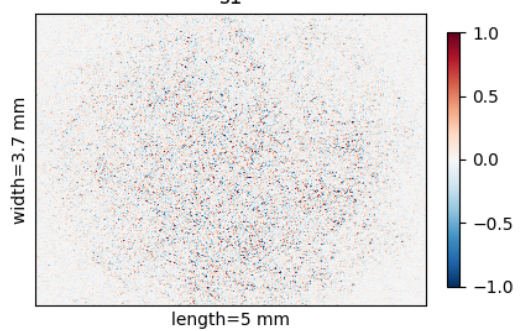

s1

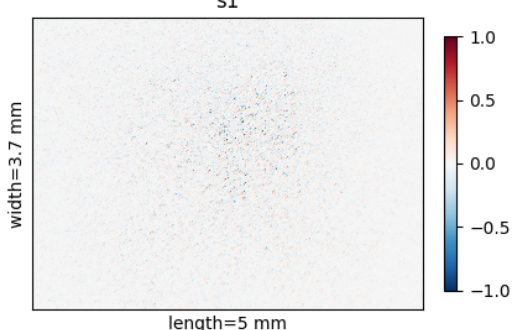

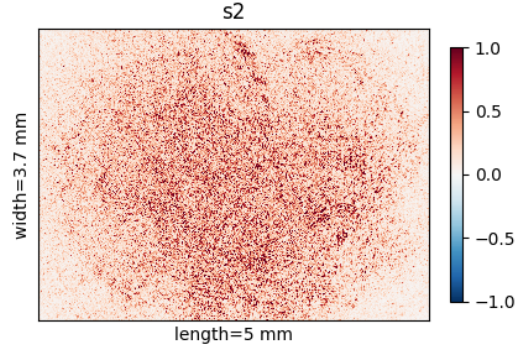

s2

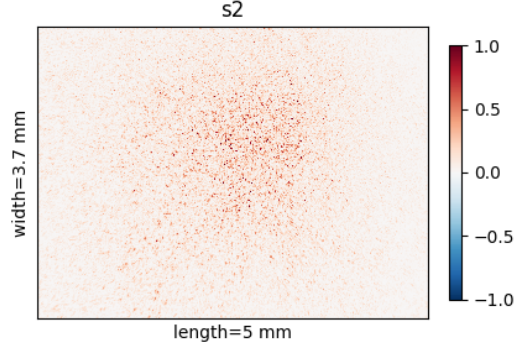

Figure 4. Experimental Stokes parameters. First row: angle of incidence $0^{\circ}$. Second row: angle of incidence $45^{\circ}$.

\section{DISCUSSION}

In the present communication, we verified that diffusers are able to modify the state of polarization of the incident light. This effect, in combination with the ability of phase encoding optical codes, might be used in optical security problems. We performed ray tracing calculations and optical experiments in order to evaluate how important the changes in polarization are. Both numerical and experimental results present a good agreement.

\section{ACKNOWLEDGMENTS}

A.C. acknowledges support from Ministerio de Economía y Competitividad (MINECO) grant number FIS201675147-C3-1-P. The authors thank Prof. Joan Esteve (Department of Applied Physics, UB) for the profilometry measurement of the diffuser.

\section{REFERENCES}

1. Goodman, J. W., [Statistical optics], John Wiley \& Sons (2015).

2. Allardyce, K. J. and George, N., "Diffraction analysis of rough reflective surfaces," Appl. Opt. 26(12), 23642375 (1987). 
3. Schertler, D. J. and George, N., "Uniform scattering patterns from grating-diffuser cascades for display applications," Appl. Opt. 38(2), 291-303 (1999).

4. Wadle, S. and Lakes, R. S., "Holographic diffusers: polarization effects," Opt. Eng. 33(4), $1084-1089$ (1994).

5. Carnicer, A. and Javidi, B., "Optical security and authentication using nanoscale and thin-film structures," Adv. Opt. Photonics 9(2), 218-256 (2017).

6. Carnicer, A., Hassanfiroozi, A., Latorre-Carmona, P., Huang, Y.-P., and Javidi, B., "Security authentication using phase-encoded nanoparticle structures and polarized light," Opt. Lett. 40(2), 135-138 (2015).

7. Carnicer, A., Arteaga, O., Pascual, E., Canillas, A., Vallmitjana, S., Javidi, B., and Bertran, E., "Optical security verification by synthesizing thin films with unique polarimetric signatures," Opt. Lett. 40(22), 5399$5402(2015)$.

8. Carnicer, A., Arteaga, O., Suñé-Negre, J. M., and Javidi, B., "Authentication of gold nanoparticle encoded pharmaceutical tablets using polarimetric signatures," Opt. Lett. 41(19), 4507-4510 (2016).

9. Markman, A., Carnicer, A., and Javidi, B., "Security authentication with a three-dimensional optical phase code using random forest classifier," J. Opt. Soc. Am. A 33(6), 1160-1165 (2016). 